\title{
BMC Nursing reviewer acknowledgement 2014
}

\author{
Catia Cornacchia
}

Masoud Amiri

Netherlands

Ergul Aslan

Turkey

Mohammadkarim Bahadori

Iran

James Balmford

Australia

Jean Barry

Switzerland

Sandra Bassendowski

Canada

\section{Linda Baumann}

USA

Dimitri Beeckman

Belgium

Carol Bennett

Canada

Linda Beuscher

USA

Melanie Bish

Australia

Joyce Black

USA

Nicole Blay

Australia

Sandy Braaf

Australia

Robyn Cant

Australia

Pierre Cardinal

Canada

\author{
Roy Carr-Hill \\ UK \\ Kristin Carson \\ Australia \\ Gianluca Catania \\ Italy \\ Ching-Min Chen \\ Taiwan \\ James Church \\ USA \\ Pamela Clarke \\ USA \\ Simon Cooper \\ Australia \\ Debra Creedy \\ Australia \\ Greta Cummings \\ Canada \\ Laura Damschroder \\ USA \\ Daniela D'Angelo \\ Italy \\ Ulrike Dapp \\ Germany \\ Rene Day \\ Canada \\ Maura Dowling \\ Ireland \\ Marc Du Bois \\ Belgium \\ Renee Du Toit \\ South Africa
}

Nuhad Dumit
Lebanon

Nancy Dunton

USA

Susan Dyess

USA

Georgios Efstathiou

Cyprus

Michaela Eikermann

Germany

Özgül Erol

Turkey

Mohammad Esmaeili Abdar

Iran

Mohammad Esmaeilpour Bandboni Iran

Ali Fakhr-Movahedi

Iran

Deirdre Fetherstonhaugh

Australia

Helen Franks

UK

Hiroki Fukahori

Japan

Martha Funnell

USA

Peter Gallagher

Nigeria

Robyn Gallagher

Australia

Laurent Gerbaud

France

Correspondence: Catia.Cornacchia@biomedcentral.com

BioMed Central, Floor 6, 236 Gray's Inn Road, London WC1X 8HB, UK 
Charlie Goldsmith

Canada

Helen Goodman

UK

Laura Goodwin

UK

Sue Green

UK

Rosane Griep

Brazil

Ruud Halfens

Netherlands

Wendy Hall

Canada

Catherine Hankey

UK

Tove Hanssen

Norway

Ian Harris

Australia

Gørill Haugan

Norway

Cornelia Heinze

Germany

Amanda Henderson

Australia

Ingela Henoch

Sweden

Mary Hickson

UK

Karen Hoare

New Zealand

Nancy Hogan

USA

Wiliam Holzemer

USA

Doris Howell

Canada

Suh-Ing Hsieh

Taiwan

Robbert Huijsman

Netherlands

Marie Hutchinson

Australia
Jeanette Ignacio

Singapore

Sedigheh Iranmanesh

Iran

David Jolley

UK

Chi-Wen Juan

Taiwan

Sharon Kaasalainen

Canada

Eulalia Kahwa

Jamaica

Mari Kangasniemi

Finland

Maria Karanikola

Cyprus

Judith Karshmer

USA

Sultan Kav

Turkey

Daniel Kelly

UK

Robin Kennedy

UK

Carole Kenner

USA

Michael Kerr

Canada

Sharon Kinney

Australia

Helen Kirkpatrick

Canada

Ruth M. Kleinpell

USA

Serena Koh

Singapore

Rachel Kornhaber

Australia

Veronica Lambert

Ireland

Kylie Lange

Australia

Heather Laschinger

Canada
Katherine Laux Kaiser

USA

I-Chuan Li

Taiwan

Sok Ying Liaw

Singapore

Violeta Lopez

Singapore

Marta Losa Iglesias

Spain

Andrew Lovell

UK

Lee-Fay Low

Australia

Tobias Luck

Germany

Marinha Sofia Macedo

Saudi Arabia

Sandra Mackey

Australia

Salvatore R Maddi

USA

Ganga Mahat

USA

Moira Maley

Australia

Sumaira Malik

UK

Anastasia Mallidou

Canada

Judy Mannix

Australia

Anna Marchetti

Italy

Wanda Martin

Canada

Randi Martinsen

Norway

Anne Matthews

Ireland

Neda Mehrdad

Iran

Anastasios Merkouris

Cyprus 
Patricia R Messmer

USA

Patriek Mistiaen

Netherlands

Yoshifumi Miyazaki

Japan

Ralph Möhler

Germany

Zena Moore

Ireland

Wendy Moyle

Australia

Terrence Murphy

USA

Teri Murray

USA

Noureddin Nakhostin Ansari Iran

Heather Newton

UK

Patricia Nicholson

Australia

Alireza Nikbakht

Nasrabadi Iran

Nicola North

New Zealand

Robert Olson

Canada

Linda O'Mara

Canada

Daniel O'Neill

USA

Eme Owoaje

Nigeria

Wolter Paans

Netherlands

Savvas Papacostas

Cyprus

Evridiki Papastavrou

Cyprus

Pammla Petrucka

Tanzania
Kathryn Pfaff

Canada

Jane Phillips

Australia

Barbara Polivka

USA

Davina Porock

UK

Janet Primomo

USA

Jamal Qaddumi

Palestinian Territory

Hossein Rafiei

Iran

Paul Ratanasiripong

USA

Michael Relf

USA

Anne-Sophie Rigaud

France

Jacqueline Ross

USA

Elizeus Rutebemberwa

Uganda

Ali Saleh

Jordan

Pavlos Sarafis

Greece

Stephanie Schim

USA

P Anne Scott

Ireland

Walter Sermeus

Belgium

Nick Sevdalis

UK

Abeer Shaheen

Jordan

Caroline Shuldham

UK

Maja Söderbäck

Sweden
Panayota Sourtzi

Greece

Annelie Sundler Johansson

Sweden

Susan Swider

USA

Miyuki Takase

Japan

Kirsti Torjuul

Norway

Hsiu-Min Tsai

Taiwan

Mimi Tse

Hong Kong

Sharon Tucker

USA

Mojtaba Vaismoradi

UK

Peter Hj Van Der Voort

Netherlands

Anneloes Van Staa

Netherlands

James Vardaman

USA

Marianne Wallis

Australia

Zhen Wang

USA

Caryn West

Australia

Lesley Wilkes

Australia

Emma Wilkinson

UK

Lilian Wong

Singapore

Bobbi Jo Yarborough

USA

Kourosh Zarea

Iran 